\title{
Experimental Investigations on Eco-Friendly Helium-Mist Near-Dry Wire-Cut EDM of M2-HSS Material
}

\author{
Boopathi Sampath ${ }^{1, a^{*}}$, M. Sureshkumar ${ }^{2, b}$, T. Yuvaraj ${ }^{1, c}$, D. Velmurugan ${ }^{1, d}$ \\ ${ }^{1}$ Department of Mechanical Engineering, Muthayammal Engineering College, Rasipuram, Tamil \\ Nadu 637408 \\ ${ }^{2}$ Department of Mechanical Engineering, Bannariamman Institute of Technology, \\ Sathyamangalam. Tamil Nadu 638401 \\ aboopasangee@gmail.com, bsureshkumarm@bitsathy.ac.in, cyuvaraj.t.mech@mec.edu.in, \\ dvelmurugan.d.mech@mec.edu.in
}

Keywords: Near Dry WEDM, Helium, M2-HSS, Minimum Quantity Lubrication, Taguchi Method

\begin{abstract}
In this paper, helium-assisted near-dry wire-cut electrical discharge machining (NDWEDM) method molybdenum wire has been used to reduce the environmental impact and to cut M2-HSS material. The pressurized non-reacting helium gas mixed with a small amount of water (Helium-mist) is used as the dielectric fluid to accomplish adequate cooling and flush-out debris. The new experimental setup has been developed to conduct the near-dry WEDM tests using the L9 orthogonal array of the Taguchi technique. The input parameters such as voltage (V), pulse-width (PW), pulse-interval (PI), and flow rate (F) of mixing water and output variables are the material removal rate (MRR) and surface roughness (Ra). It was observed that MRR and Ra are amplified by the rise in voltage and pulse-width, and flow rate conversely, the pulse interval minimizes the responses. The percentage of contribution of pulse width, voltage, pulse interval and flow rate are $24.06 \%, 32.98 \%, 12.75 \%$ and $30.21 \%$ on MRR and $20.94 \%, 22.22 \%, 47.86 \%$ and $8.97 \%$ on Ra respectively. Finally, the confirmation trials have been accomplished to validate the foreseen best parameter sets on optimal responses.
\end{abstract}

\section{Introduction}

In the WEDM process, manufacturing characters and environmental influence are deliberated to analyze the machining mechanics, tool change, minimum rejections in production, and effects of cutting-fluid flow[1]. The environmental effect of machining should be analyzed for minimizing environmental impacts by modification of existing technology and the development of new manufacturing methods[2]. In these aspects, research on the modification of EDM and WEDM processes was developed to make the trade-off techniques between machining performance and machining pollutions.

The many experimental research on dry and near-dry EDM processes have previously been studied to find the analytical relationship for tool wear, MRR, the influence of dielectric fluid, and environmental impacts such as toxicity and flammability[2,3]. However, the dry and near-dry WEDM process, the investigations had so far been studied to improve the cutting performances as follows. The first systematic study in near-dry WEDM was carried out to investigate the air-mist dielectric fluid to cut the HSS-M2 steel materials. The optimal parameters for best machining performance had been predicted using the evolutionary optimization method[4]. The conventional tap water missed with air as dielectric has been tried to analyze the influences on cutting factors [5]. The oxygen-mist NDWEDM process has been investigated by using the response surface method to reveal the effect of oxygen-mist in WEDM Process[6]. It was observed from comparative 
studies, the oxygen-mist near-dry WEDM process has better performance than the air-mist near-dry process [7]. The Monel metal, EN-31 die steel, ANSI-D3 steel, and Tungsten carbide (WC) as work materials and air/ gas-mist as dielectric fluids had been investigated in near-dry WEDM processes[8,9]. It was also tried to investigate the near-dry WEDM process to cut various alloy materials by few researchers. Thus, there is no attempt found in the helium-mist near-dry WEDM process to cut the HSS-M2 materials.

In this research, the helium gas -water as dielectric and molybdenum wire electrode are used as key factors to investigate the machining performance by Taguchi L9 orthogonal array. The significant factors and percentage of contributions on machining performance are discussed.

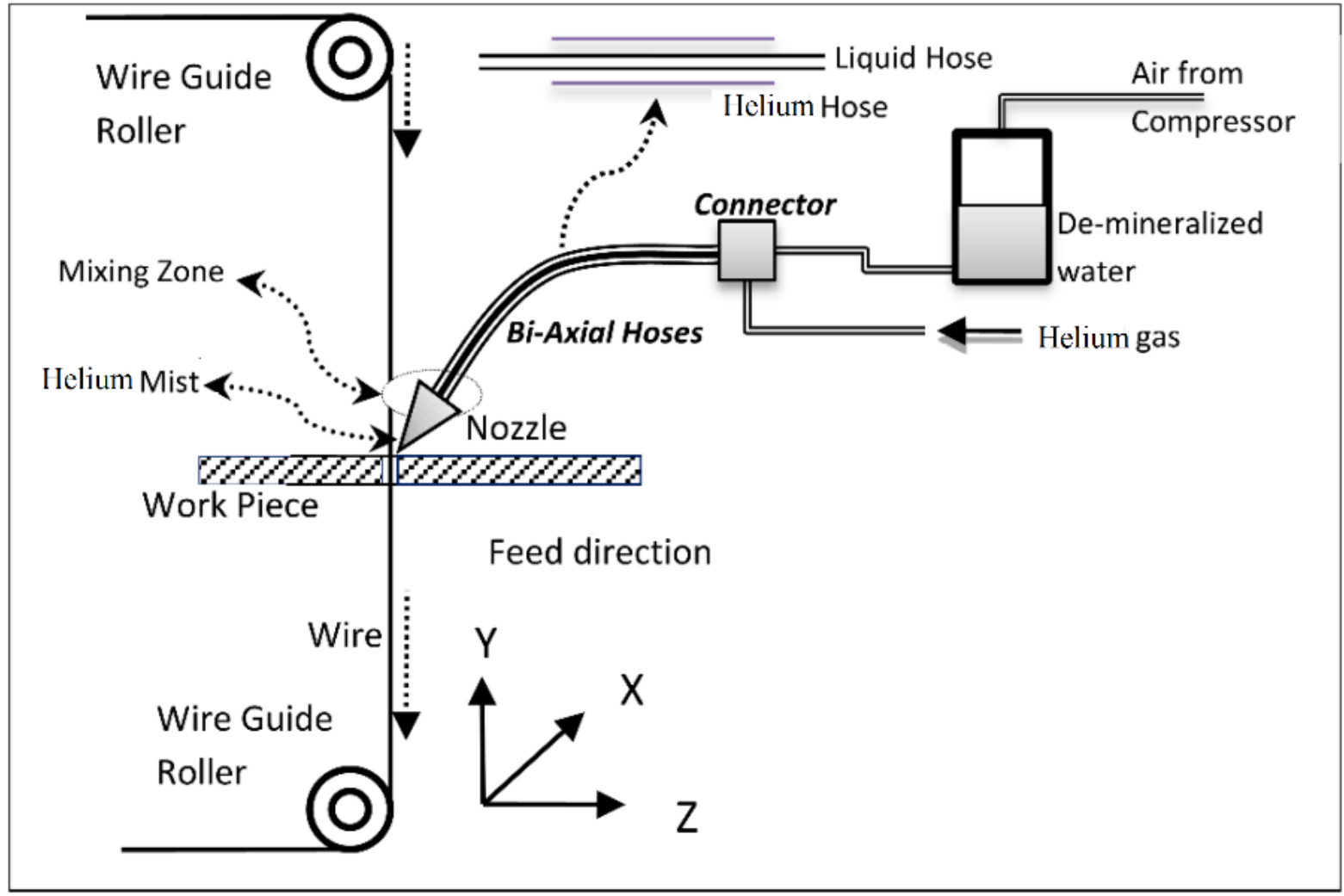

Figure 1. Experimental Setup of Helium assisted near-dry WEDM

\section{Experimental setup and Experimentation}

The $5 \mathrm{~mm}$ thickness of M2-HSS was used as work material for helium-mist near dry WEDM processes. The investigational setup of Helium-assisted near-dry WEDM is exposed in Figure 1. The helium gas was supplied outside the hose and a small quantity of water is flowing through the inner hose. Both fluids are mixed at the end of co-axial hoses to the nozzle. Helium-mist was acting as a working medium in reciprocating NDWEDM machine. The voltage, pulse width, and pulse interval can be adjusted from Fuzzy controlled WEDM. The mixing flow rate of small water was controlled and measured by a new experimental setup. The surface roughness of WEDM was directly measured along with four different passes over the workpiece surface by the roughness tester. The material removal rate (MRR) can be premeditated by the ratio of the volume of debris removed with a period using Equations (1) and (2).

Kerf $=$ wire diameter $+2 \times$ times of parking gap $=0.20 \mathrm{~mm}$ 
$M R R=($ thickness $*$ Kerf $*$ length of cut $) /$ time $\quad \mathrm{mm}^{3} / \mathrm{min}$

Based on the trial test, the inputs and their required levels are recognized. The levels of each process variable are arranged in Table 1. The experimentations are steered using the L9 orthogonal array of the Taguchi method[10] and the MRR and Ra values observed over the trials are shown in Table 2.

Table 1. Helium assisted Near-dry WEDM levels of input variables

\begin{tabular}{|c|c|c|c|c|c|}
\hline Symbol & Input factor & Units & Level 1 & Level 2 & Level 3 \\
\hline V & Gap-Voltage & $\mathrm{V}$ & 3 & 5 & 4 \\
\hline PW & Pulse width & $\mu \mathrm{s}$ & 15 & 25 & 20 \\
\hline PI & Pulse interval & $\mu \mathrm{s}$ & 45 & 75 & 60 \\
\hline F & Mixing water Flow Rate & $\mathrm{ml} / \mathrm{min}$ & 10 & 20 & 15 \\
\hline
\end{tabular}

Table 2. Experimental observation using L9 design of experiment

\begin{tabular}{|c|c|c|c|c|c|c|}
\hline \multirow{2}{*}{ S.N. } & \multicolumn{5}{|c|}{ Input factors } & \multicolumn{2}{c|}{ Mean Responses } \\
\cline { 2 - 7 } & V & PW & PI & F & MRR $\left(\mathrm{mm}^{3} / \mathrm{min}\right)$ & Ra $(\mu \mathrm{m})$ \\
\hline 1. & 3 & 15 & 45 & 10 & 4.14 & 1.17 \\
\hline 2. & 3 & 20 & 60 & 15 & 7.065 & 1.08 \\
\hline 3. & 3 & 25 & 75 & 20 & 9.414 & 1.26 \\
\hline 4. & 4 & 15 & 60 & 20 & 7.596 & 1.17 \\
\hline 5. & 4 & 20 & 75 & 10 & 6.003 & 1.08 \\
\hline 6. & 4 & 25 & 45 & 15 & 11.619 & 1.89 \\
\hline 7. & 5 & 15 & 75 & 15 & 7.281 & 1.08 \\
\hline 8. & 5 & 20 & 45 & 20 & 13.896 & 2.16 \\
\hline 9. & 5 & 25 & 60 & 10 & 9.504 & 1.53 \\
\hline
\end{tabular}

Table 3. Taguchi analysis for MRR to predict the percentage of contribution

\begin{tabular}{|c|c|c|c|c|}
\hline Input & $\begin{array}{c}\text { Degree of } \\
\text { Freedom }\end{array}$ & The sequential sum of square & $\begin{array}{c}\text { Percentage of } \\
\text { contribution }\end{array}$ & Rank \\
\hline V & 2 & 16.9154 & 24.06 & 3 \\
\hline PW & 2 & 23.1813 & 32.98 & 1 \\
\hline PI & 2 & 8.9658 & 12.75 & 4 \\
\hline F & 2 & 21.2329 & 30.21 & 2 \\
\hline Total & 8 & 70.2954 & - & - \\
\hline
\end{tabular}

Table 4. Taguchi analysis for Ra to predict the percentage of contribution

\begin{tabular}{|c|c|c|c|c|}
\hline Input & $\begin{array}{c}\text { Degree of } \\
\text { Freedom }\end{array}$ & The sequential sum of square & $\begin{array}{c}\text { Percentage of } \\
\text { contribution }\end{array}$ & Rank \\
\hline V & 2 & 0.2646 & 20.94 & 3 \\
\hline PW & 2 & 0.2808 & 22.22 & 1 \\
\hline PI & 2 & 0.6048 & 47.86 & 4 \\
\hline F & 2 & 0.1134 & 8.97 & 2 \\
\hline Total & 2 & 1.2636 & - & - \\
\hline
\end{tabular}




\section{Result analysis}

Taguchi's analysis for MRR and $\mathrm{Ra}$ are shown in Tables 3 and 4 respectively. The contribution (\%) of gap voltage, pulse width, pulse interval, and flow rate on MRR is $24.06 \%, 32.98 \%, 12.75 \%$, and $30.21 \%$ respectively. Similarly, the percentage of contribution of gap voltage, pulse width, pulse interval, and flow rate on $\mathrm{Ra}$ is $20.94 \%, 22.22 \%, 47.86 \%$, and $8.97 \%$ respectively. The pulse width is the most significant factor on MRR due to an increase in spark strength[4,6]. The flow rate is the second important factor on both MRR and Ra due to provide sufficient cooling in the plasma zone and flush out debris. The pulse interval is the more dominant factor on surface roughness due to discrete spark by long pulse pause time[5,11]. While increasing MRR by controlling factors, the surface roughness also getting increased due to the coarse of debris. The percentage of contributions of input variables on MRR and Ra are illustrated in Figure 2.

\section{Percentage of Contribution on MRR and Ra}

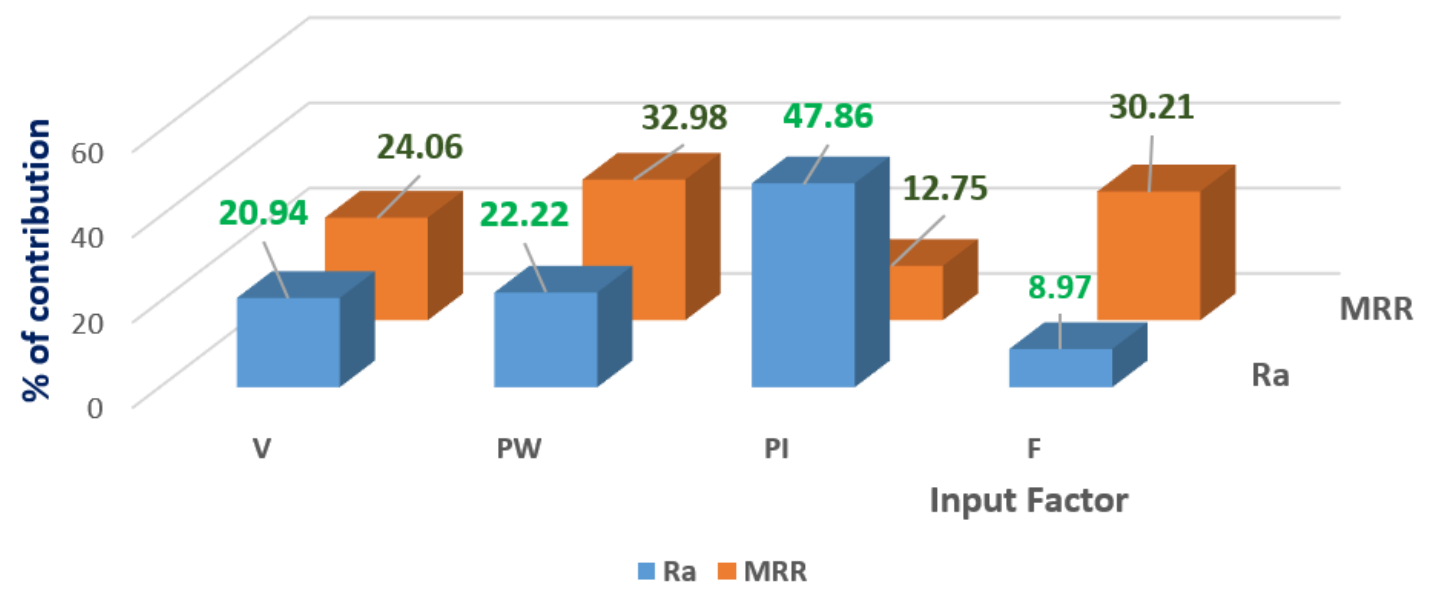

Figure 2. Input factors Contributions on Machining Responses

Figures 3(a) \&(b) are illustrating the mean effects on the MRR and Ra respectively. The plots show that the MRR and Ra are significantly amplified due to the growth in gap voltage, pulse width, and flow rate. Conversely, MRR and $\mathrm{Ra}$ values are condensed by growing pulse interval[4,5]. Taguchi analysis tests and the percentage of contribution of process factors are illustrated for MRR and $\mathrm{Ra}$ are shown in Tables 3 and 4 respectively. It was revealed that pulse-width is a very significant contribution to MRR and pulse interval has more contribution on $\mathrm{Ra}$. The plots show that voltage, pulse width, and flow rate have a more momentous consequence on MRR and Ra than pulse-interval[8,9,12-14]. The validation tests are used to authorize the predicted outcomes from the Taguchi Method. The proof tests are completed by steering the experimentations with an exact influence of the levels as shown in Table 5. The following Equation (3) is applied to estimate the best of the MRR and $\mathrm{Ra}[4]$.

$$
\delta_{o p t}=\delta_{\text {tot }}+\sum_{j=1}^{n}\left(\delta_{j}-\delta_{\text {tot }}\right)
$$

Table 5. Validation of Taguchi Prediction results.

\begin{tabular}{|c|c|c|c|c|c|c|c|}
\hline \multirow{2}{*}{ Response } & \multicolumn{4}{|c|}{ Process Parameters } & \multirow{2}{*}{$\begin{array}{l}\text { Predicted } \\
\text { Value }\end{array}$} & \multirow{2}{*}{$\begin{array}{c}\text { Experiment } \\
\text { Value }\end{array}$} & \multirow[t]{2}{*}{ Unit } \\
\hline & $\mathrm{V}$ & PW & PI & $\mathrm{F}$ & & & \\
\hline MRR & 5 & 25 & 45 & 20 & 15.08 & 15.45 & $\mathrm{~mm}^{3} / \mathrm{min}$ \\
\hline $\mathrm{Ra}$ & 3 & 15 & 75 & 10 & 0.57 & 0.60 & $\mu \mathrm{m}$ \\
\hline
\end{tabular}


The best response values are predicted using the Taguchi technique using the trial version of MINITAB ${ }^{\circledR}$ software. The input factor combination $\left(\mathrm{V}_{3} \mathrm{PW}_{3} \mathrm{PI}_{1} \mathrm{~F}_{3}\right)$ for the maximization of MRR is shown in Figures 2(a). Similarly, the input factor combination $\left(\mathrm{V}_{1} \mathrm{PW}_{1} \mathrm{PI}_{3} \mathrm{~F}_{1}\right)$ for minimization of is Ra showed in Figures 2(b). The maximum of MRR $\left(15.09 \mathrm{~mm}^{3} / \mathrm{min}\right)$ has been found with the grouping of input factors of $\mathrm{V}_{3} \mathrm{PW}_{3} \mathrm{PI}_{1} \mathrm{~F}_{3}$ from the validation test. Similarly, the smallest value of $\mathrm{Ra}(0.57 \mu \mathrm{m})$ has been found with the grouping of input factors of $\mathrm{V}_{1} \mathrm{PW}_{1} \mathrm{PI}_{1} \mathrm{~F}_{3}$ by evaluation test. While increasing pulse width and gap voltage, the MRR and Ra are maximized due to the high intensity of spark between wire and work material[6]. The highest MRR and Ra are achieved by the maximum flow rate of the dielectric medium due to quick flushing of debrides components[15].
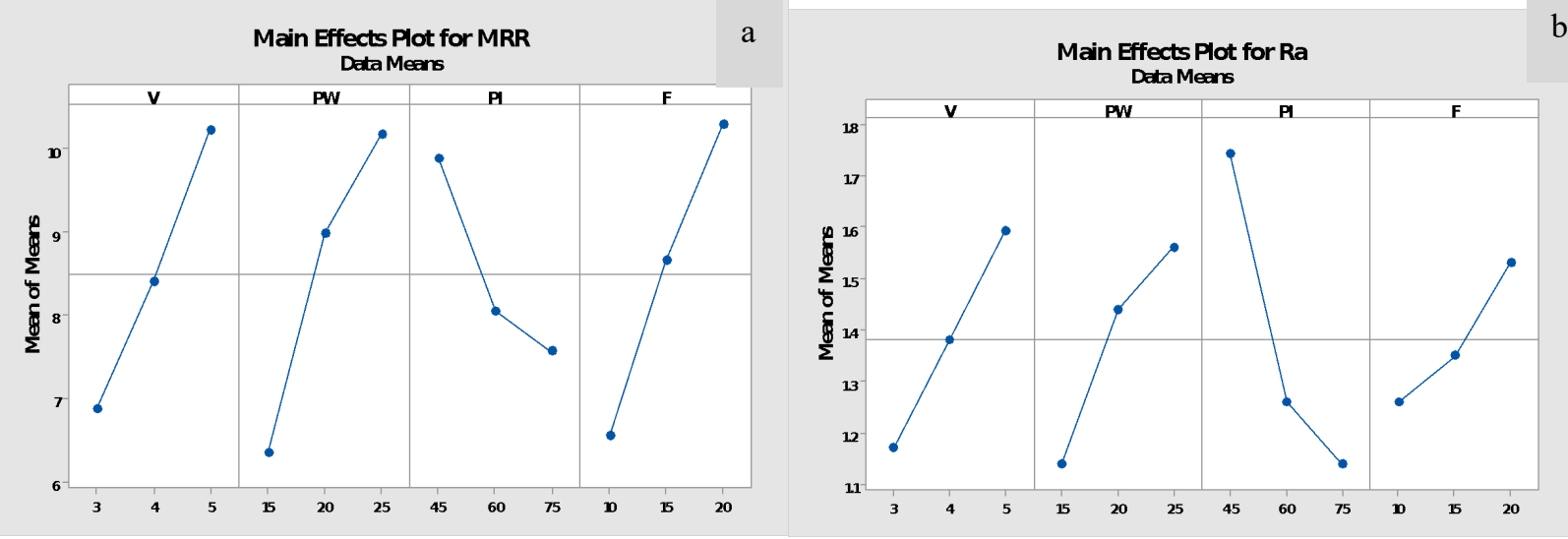

Figure 3 Input factor's influences on (a) MRR (b) Ra

\section{Conclusions}

The new helium-mist Near-dry WEDM experiments were conducted to cut M2-HSS material and estimate the optimal process variables for machining responses. As per the Taguchi analysis, the following conclusions were obtained.

- The percentage of contribution of gap voltage, pulse width, pulse interval, and flow rate on MRR is $24.06 \%, 32.98 \%, 12.75 \%$, and $30.21 \%$ respectively. Similarly, the percentage of contribution of gap voltage, pulse width, pulse interval, and flow rate on $\mathrm{Ra}$ is $20.94 \%$, $22.22 \%, 47.86 \%$, and $8.97 \%$ respectively.

- The maximum of MRR $\left(15.09 \mathrm{~mm}^{3} / \mathrm{min}\right)$ has been found with the grouping of input factors of $\mathrm{V}_{3} \mathrm{PW}_{3} \mathrm{PI}_{1} \mathrm{~F}_{3}$ from the validation test. Similarly, the smallest value of $\mathrm{Ra}$ $(0.57 \mu \mathrm{m})$ has been found with the grouping of input factors of $\mathrm{V}_{1} \mathrm{PW}_{1} \mathrm{PI}_{3} \mathrm{~F}_{1}$ by evaluation test.

- While increasing gap voltage, pulse width, and flow rate the MRR and Ra are maximized due to the high intensity of heat between the wire and work material. The highest MRR and $\mathrm{Ra}$ are found by the maximum flow rate of the dielectric medium due to quick flushing of debrides components. However, the $\mathrm{Ra}$ is proportionally increased with increasing MRR.

- The poor MRR and Ra were obtained by increasing pulse interval due to the expansion of spark-free time. 


\section{References}

[1] A.A. Munoz, P. Sheng, An analytical approach for determining the environmental impact of machining processes, J. Mater. Process. Tech., 53 (1995) 736-758. https://doi.org/10.1016/0924-0136(94)01764-R

[2] S.H. Yeo, H.C. Tan, A.K. New, Assessment of waste streams in electric-discharge machining for environmental impact analysis, Proc. Inst. Mech. Eng. Part B J. Eng. Manuf., 212 (1998) 393400. https://doi.org/10.1243/0954405981515996

[3] M.S. Hewidy, T.A. El-Taweel, M.F. El-Safty, Modelling the machining parameters of wire electrical discharge machining of Inconel 601 using RSM, J. Mater. Process. Technol., 169 (2005) 328-336. https://doi.org/10.1016/j.jmatprotec.2005.04.078

[4] S. Boopathi, K. Sivakumar, Experimental investigation and parameter optimization of near-dry wire-cut electrical discharge machining using multi-objective evolutionary algorithm, Int. J. Adv. Manuf. Technol., 67 (2013) 2639-2655. https://doi.org/10.1007/s00170-012-4680-4

[5] S. Boopathi, K. Sivakumar, Study of water assisted dry wire-cut electrical discharge machining, Indian J. Eng. Mater. Sci., 21 (2014) 75-82.

[6] S. Boopathi, K. Sivakumar, Optimal parameter prediction of oxygen-mist near-dry Wire-cut EDM, Int. J. Manuf. Technol. Manag., 30 (2016) 164-178. https://doi.org/10.1504/IJMTM.2016.077812

[7] S. Boopathi, K. Sivakumar, Experimental comparative study of near-dry wire-cut electrical discharge machining (WEDM), Eur. J. Sci. Res., 75 (2012) 472-481.

[8] S. Boopathi, S. Myilsamy, Material removal rate and surface roughness study on Near-dry wire electrical discharge Machining process, Mater. Today Proc., xx (2021) xx-xx. https://doi.org/10.1016/j.matpr.2021.02.267

[9] Y. Jia, B.S. Kim, D.J. Hu, J. Ni, Parametric study on near-dry wire electrodischarge machining of polycrystalline diamond-coated tungsten carbide material, Proc. Inst. Mech. Eng. Part B J. Eng. Manuf., 224 (2010) 185-193. https://doi.org/10.1243/09544054JEM1602

[10] S. Boopathi, Experimental investigation and parameter analysis of LPG refrigeration system using Taguchi method, SN Appl. Sci., 1 (2019) 892. https://doi.org/10.1007/s42452-019-0925-2 [11] S.K. Saha, S.K. Choudhury, Experimental investigation and empirical modeling of the dry electric discharge machining process, Int. J. Mach. Tools Manuf., 49 (2009) 297-308. https://doi.org/10.1016/j.ijmachtools.2008.10.012 [12] C.C. Kao, J. Tao, S. Lee, A.J. Shih, Dry wire electrical discharge machining of thin workpiece, in: Trans. North Am. Manuf. Res. Inst. SME, 2006: pp. 253-260.

[13] C.C. Kao, J. Tao, A.J. Shih, Near dry electrical discharge machining, Int. J. Mach. Tools Manuf., 47 (2007) 2273-2281. https://doi.org/10.1016/j.ijmachtools.2007.06.001

[14] S. Abdulkareem, A.A. Khan, M. Konneh, Reducing electrode wear ratio using cryogenic cooling during electrical discharge machining, Int. J. Adv. Manuf. Technol., 45 (2009) 11461151. https://doi.org/10.1007/s00170-009-2060-5

[15] S. Boopathi, Experimental Comparative Study of Near-Dry Wire-Cut Electrical Discharge Machining ( WEDM ), 75 (2012) 472-481. 